\title{
Tumour budding : an emerging prognostic factor in colorectal carcinoma
}

\author{
Bimalka Seneviratne, Bawantha Gamage \\ Faculty of Medical Sciences, University of Sri Jayewardenepura, Sri Lanka
}

Key words: Equitable care; endocrine surgery

\begin{abstract}
Tumour budding, is a morphological feature seen in colorectal carcinoma and known to be associated with a poor outcome. It is thought to be representing the phenomenon of epithelial-mesenchymal transition of colorectal carcinoma. Colorectal malignancies exhibiting budding have shown an association with lymphatic invasion and lymph node metastasis. In addition tumour budding has proven to be associated with tumour recurrence and is a negative prognostic indicator of overall survival of patients with colorectal carcinoma.
\end{abstract}

Objectives of the study was to assess the prevalence of tumour budding in CRC (higher colorectal carcinoma) specimens and to determine the association between tumour budding and the histological grade, nodal involvement and tumour recurrence.

A retrospective study was carried out at the Department of Pathology, Faculty of Medical Sciences, University of Sri Jayewardenepura. The study sample included 58 cases of colorectal carcinoma during the period January 2015 to June 2018. The histological grade, nodal status and tumour recurrence was recorded following review of histology slides. In addition, the slides were examined for the presence or absence of tumour budding, according to the ITBCC (November 2016) recommendations.

Study population showed a tumour budding percentage of $32.75 \%$. Tumour budding in colorectal carcinoma showed a statistically significant association with high histological grade, lymph nodal involvement and tumour recurrence ( $p$ value $<0.05)$.

Tumour budding in CRC is strongly predictive of high histological grade, lymph node metastases, and tumour recurrence. The study population included 02 cases of tumour recurrence in the budding group, and a single case of

\footnotetext{
Correspondence: Bawantha Gamage

E-mail: bawantha@sjp.ac.lk

Received: 15-11-2018 Accepted: 27-12-2018

(iD) http://orcid.org/0000-0003-4381-228X DOI: http://doi.org/10.4038/sljs.v36i4.8551
}

cancer related deaths.

\section{Introduction}

Tumour budding which is being widely discussed in many centres, is likely to be emerging as a novel prognostic marker in colorectal carcinoma [1]. Numerous studies have shown the clinical significance of tumour budding as an independent risk factor which is associated with an adverse outcome [2].

Tumour budding is assessed at the invasive front of colorectal carcinoma. The presence of budding is confirmed by finding of detached tumour cells either singly or in small clusters within the surrounding stroma [3]. Tumour budding, is a histomorphological biomarker associated with an adverse outcome. It represents de-differentiation of epithelial cells into more aggressive phenotypes (figure 1,2). Tumour budding in colorectal carcinoma has shown to be significantly associated with lymphatic invasion and lymph node metastases [4]. In addition it is a negative prognostic indicator in relation to overall survival. Inclusion of this morphological parameter into the CRC staging algorithm may be clinically relevant, but will require consensus opinion on the pathological description of tumour budding [5].

In view of arriving at a consensus opinion a panel of experts discussed the issues related to standardized reporting of tumor budding in colorectal carcinoma, at the International Tumour Budding Consensus Conference (ITBCC) held in Bern, Switzerland, in November 2016.

The primary goal of the ITBCC was to reach agreement on an international, evidence-based and reproducible scoring system for histological reporting of tumor budding [6]. The definition, evaluation, severity and interpretation of tumour budding were some of the key areas discussed in detail at this conference [7] Recommendations of the ITBCC, which achieved consensus have been incorporated into the CAP cancer protocol for the handling of specimens of colorectal carcinoma.

There were total of 11 recommendations of the ITBCC. From the total of 11 recommendations, ten were able to achieve 100 $\%$ agreement from the panel members. One recommendation 
was agreed upon by $96 \%$ (No. 5 below) of the panelists.

The suggestions 1, 5, 7 through 9, and 11 have been included into the CAP cancer protocol as the recommended method for assessing tumour budding in colorectal carcinoma. Further to this the Royal College of Pathologists of the United Kingdom (V4, December 2017) has acknowledged ITBCC in pathology reporting guidelines for colorectal carcinoma. To determine tumour budding it is mandatory to examine the entire invasive front of the tumour under the light microscope. A single "hot spot" needs to be selected after carefully assessing the hematoxylin \& eosin stained tissue sections of the invasive front (defined as single tumour cells or clusters of up to 4 tumour cells), with an $\times 20$ objective lens. As the micros-copic field diameters vary, the application of a correction factor has been suggested to report the number of tumour buds in the equivalent of a $0.785-\mathrm{mm} 2$ field. The tumour bud score has been determined by using a 3 -tiered system based on the number of tumour buds in a 0.785-mm2 field (low, 0-5 tumour buds; intermediate, 6-9 tumour buds; high, 10 or more tumour buds).

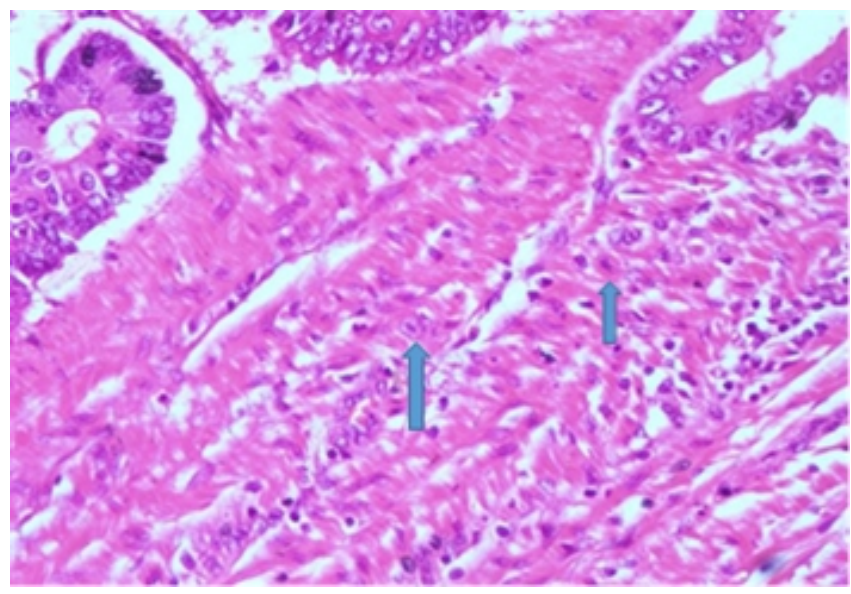

Figure 1. Moderately-differentiated adenocarcinoma of rectum exhibiting tumour budding (detached tumour cells at the advancing edge-arrows). H\&E stain x 100
However, it is widely acknowledged that additional studies will be required to address the challenges in uniform reporting of tumour budding [8].

\section{Objectives}

To assess the prevalence of tumour budding in CRC specimens and determine the association between tumour budding and the histological grade, nodal involvement and tumour recurrence.

\section{Methodology}

A retrospective study was carried out at the Department of Pathology, Faculty of Medical Sciences, University of Sri Jayewardenepura. The study sample included 58 cases of colorectal carcinoma during the period January 2015 to June 2018. Specimens that have been handled and processed according to the standard guidelines were included in the study group. Following tissue processing, $3 \mu \mathrm{m}$ thick sections have been prepared for staining with the routine hematoxylin and eosin method.

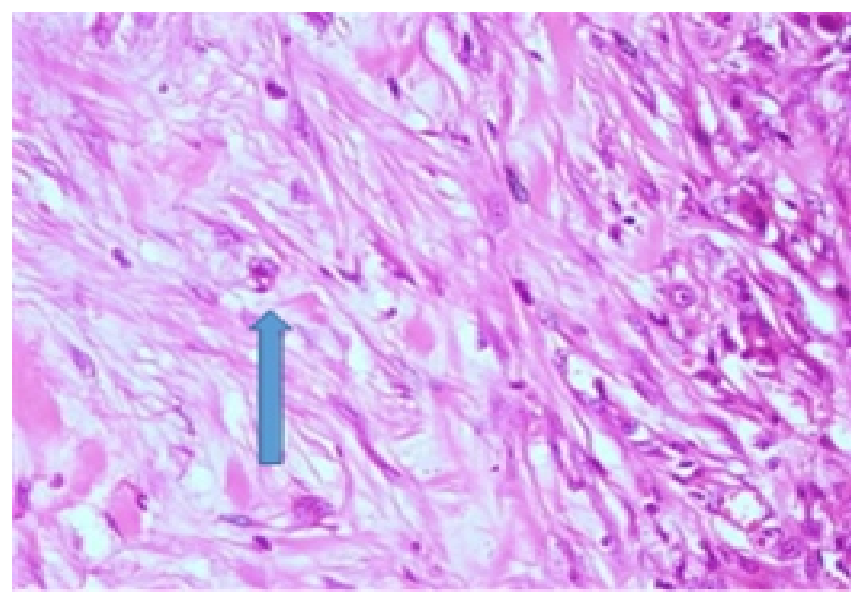

Figure 2. Single cells (tumour budding) at the advancing edge of moderately differentiated adenocarcinoma of rectum (arrows). H\&E stain x 100

Recommendations of the ITBCC are as follows;

\begin{tabular}{|l|l|}
\hline 1. & Tumour budding is defined as a single tumour cell or a cell cluster of up to 4 tumour cells. \\
\hline 2. & Tumour budding is an independent predictor of lymph node metastasis in pT1 colorectal cancer. \\
\hline 3. & Tumour budding is an independent predictor of survival in stage II colorectal cancer. \\
\hline 4. & $\begin{array}{l}\text { Tumour budding should be taken into account along with other clinicopathologic factors in a multidisciplinary } \\
\text { setting. }\end{array}$ \\
\hline 5. & Tumour budding is counted on hematoxylin-eosin ( $\mathrm{H} \& \mathrm{E})$. \\
\hline 6. & Intratumoral tumour budding in colorectal cancer has been shown to be related to lymph node metastasis. \\
\hline 7. & Tumour budding is assessed in 1 hotspot (in a field measuring $0.785 \mathrm{~mm}^{2}$ ) at the invasive front. \\
\hline 8. & For tumour budding assessment in colorectal cancer, the hotspot method is recommended. \\
\hline 9. & A 3 -tier system should be used along with the budding count to facilitate risk stratification in colorectal cancer. \\
\hline 10. & Tumour budding should be included in guidelines/protocols for colorectal cancer reporting. \\
\hline 11. & Tumour budding and tumour grade are not the same. \\
\hline
\end{tabular}

The Sri Lanka Journal of Surgery 2018; 36(4): 1-5 
Poor quality slides were re-stained, and the histological diagnoses of all the cases were reviewed independently by two pathologists and confirmed. Prognostic information such as tumour grade and lymph nodal status were also recorded, after examining the histology slides. In addition, the slides were examined for the presence or absence of tumour budding, according to the ITBCC (November 2016) recommendations. Data was tabulated without revealing the identification details. Information with regard to tumour recurrence was extracted from the data base.

\section{Inclusion criteria:}

- Colorectal carcinoma specimens during the study period, from January 2015 to June 2018, in which the histology slides and paraffin blocks were available.
Exclusion criteria:

- Colorectal carcinoma specimens during the study period (January 2015 to June 2018), with no clinical details.

- Colorectal carcinoma specimens during the above period with no available histology slides or paraffin blocks.

\section{Discussion}

Study population showed a tumour budding percentage of $32.75 \%$ (table 1). Tumour budding in colorectal carcinoma showed a statistically significant association ( $p$ value $<$ 0.05 ) with high histological grade (poorly differentiated), lymph nodal involvement, and tumour recurrence (distant).

The results of the research have been similar to multiple studies done in tertiary referral centres in other parts of the globe, although the smaller sample size of the current study

\section{Results}

\begin{tabular}{|c|c|c|c|c|c|}
\hline Tumour budding & Grade & Stage (TNM) & $\begin{array}{l}\text { Lymph nodal } \\
\text { involvernent }\end{array}$ & $\begin{array}{l}\text { Completeness of resection } \\
\text { (circumferential resection } \\
\text { margin) }\end{array}$ & Recurrence \\
\hline $\begin{array}{l}\text { Tumour budding }(+) \\
n=19(32.75 \%)\end{array}$ & $\begin{array}{l}\text { High - } 16 \\
\text { Low - } 03\end{array}$ & $\begin{array}{ll}\text { I- } & 02 \\
\text { IIA- } & 04 \\
\text { IIB- } & 00 \\
\text { IIC- } & 00 \\
\text { IIIA- } & 01 \\
\text { IIIB- } & 12 \\
\text { IIIC- } & 00 \\
I V- & 00\end{array}$ & $\begin{array}{l}\text { present }-13 \\
\text { absent - } 03\end{array}$ & $\begin{array}{l}\text { CRM - uninvolved } \\
(>2 \mathrm{~mm})\end{array}$ & $\begin{array}{l}\mathrm{n}=02 \\
\text { (distant) }\end{array}$ \\
\hline $\begin{array}{l}\text { Tumour budding (-) } \\
n=39(67.24 \%)\end{array}$ & $\begin{array}{l}\text { High }-27 \\
\text { Low - } 12\end{array}$ & $\begin{array}{ll}\text { I- } & 04 \\
\text { IIA- } & 22 \\
\text { IIB- } & 04 \\
\text { IIC- } & 00 \\
\text { IIIA- } & 04 \\
\text { IIIB- } & 05 \\
\text { IIIC- } & 00 \\
\text { IV- } & 00\end{array}$ & $\begin{array}{l}\text { present }-07 \\
\text { absent - } 32\end{array}$ & $\begin{array}{l}\text { CRM-uninvolved } \\
(>2 \mathrm{~mm})\end{array}$ & $n=0$ \\
\hline
\end{tabular}

\begin{tabular}{|c|c|c|c|c|c|c|}
\hline $\begin{array}{l}\text { Tumour } \\
\text { budding vs }\end{array}$ & $\begin{array}{l}\text { True } \\
\text { Positives }\end{array}$ & All positives & PPV \% & $\begin{array}{l}\text { True } \\
\text { Negatives }\end{array}$ & All Negatives & NPV\% \\
\hline $\begin{array}{l}\text { High-grade } \\
\text { tumours }\end{array}$ & 16 & $\begin{array}{l}35 \\
\text { (all high - grade) }\end{array}$ & 45.71 & 03 & $\begin{array}{l}23 \\
\text { (all low-grade) }\end{array}$ & 13.04 \\
\hline $\begin{array}{l}\text { Low-grade } \\
\text { tumours }\end{array}$ & 3 & $\begin{array}{l}23 \\
\text { (all low-grade) }\end{array}$ & 13.04 & 16 & $\begin{array}{l}35 \\
\text { (all high-grade) }\end{array}$ & 45.71 \\
\hline $\begin{array}{l}\text { Lymph nodal } \\
\text { involvement }\end{array}$ & 13 & 19 & 68.42 & 06 & 39 & 15.38 \\
\hline Recurrence & 02 & 02 & 100 & 17 & 56 & 30.35 \\
\hline
\end{tabular}


was considered as a major limiting factor [9]. A multicentre study with the participation of a larger number of patients from different regions, will be the next step to evaluate the impact of tumour budding in a Sri Lankan perspective.

Collaborative studies conducted by Ishikawa Y, AishimaFukasawa $\mathrm{Y}$ et al [10], has shown a significant association between tumour budding and lymphatic invasion thus leading to lymph node involvement.

More comprehensive studies done in a larger cohort of patients by Lugli et al [2], Van Wyk HC et al [5], have shown that tumour budding is a negative prognostic indicator in relation to tumour recurrence and overall survival of CRC patients. Studies done by Choi JY et al [11], and Di Gregorio $\mathrm{C}$ et al [12] have been able to highlight the association of tumour budding and distant metastasis.

Reserach done by Glasgow SC et al and Mou S et al further strengthened the concept of tumour budding as an emerging prognostic factor of colorectal carcinoma $[13,14]$.

Multicentre studies concluded by Petrelli F [15] and Rogers AC [16] also have emphasized the adverse relationship between tumour budding and colorectal carcinoma.

The TNM staging system for colorectal carcinoma separates patients in to different categories. Management of patients to a great extent depends on the stage of the disease. However, there is significant diversity in relation to the prognosis among the patients with CRC of a similar stage [17]. Currently there is no superior method of staging which has been validated [18]. The future of CRC will depend on the identification of novel, clinically relevant prognostic markers which would have an impact on the disease free interval and cancer related deaths. Better correlation with the prognostic markers would enable to reduce under and over treatment of patients with CRC. Treatment related parameters have the potential to revolutionize the outcome of colorectal carcinoma [19].

In spite of all the current information tumour budding has not been included in the TNM staging of colorectal carcinoma, largely due to the lack of consensus with regard to its definition and method of detection in routine histology sections. Many studies have addressed the issue of "reproducibility" of the assessment method and results [20]. Another concern when trying to incorporate tumour budding in to clinical practice is the lack of a suitable method of detection for all stages of CRC [21]. Some methods for reporting refer to early stages (I, II) and some are for advanced disease [22].
However, those tumours exhibiting budding certainly are worth considering as a subset to target with adjuvant chemotherapy in CRC as a whole (xiv, xv). Results would certainly have beneficial effects on long term survival of patients with colorectal carcinoma.

\section{Conclusion}

Tumour budding in CRC shows significant association with high histological grade, lymph nodal involvement and recurrence. The study population included 02 cases of tumour recurrence in the budding group, and a single case of cancer related deaths, despite the smaller sample size which was considered as a major limiting factor. Tumour budding has been studied predominantly at the invasive front of the tumour and is referred to as "peritumoral budding". Inclusion of this histological finding into the CRC staging algorithm is quite necessary, but will require consensus opinion \& standardization of the pathological definition of tumour budding [11]. Routine reporting of tumour bud count and severity is now being recommended. There is considerable interobserver variability with regard to the reporting of tumour budding which could be streamlined in the near future with the results of more comprehensive research $[12,13]$ and meta-analyses. Tumour budding has been incorporated in to the CAP (College of American Pathologists) protocol as a recommended element. Inclusion in to the TNM stage of CRC would require more collaborative research data, but certainly would be an option that has to be considered in relation to the future management of patients with colorectal carcinoma.

All authors disclose no conflict of interest. The study was conducted in accordance with the ethical standards of the relevant institutional or national ethics committee and the Helsinki Declaration of 1975, as revised in 2000 .

\section{References}

1. Koelzer VH, Zlobec I, Lugli A. Tumor budding in colorectal cancer-ready for diagnostic practice? Hum Pathol. 2016; 47 (1): 4-19. https://doi.org/10.1016/j.humpath.2015.08.007

2. Lugli A, Karamitopoulou E, Zlobec I. Tumour budding: a promising parameter in colorectal cancer. Br J Cancer. 2012; 106 (11): 1713-1717. https://org/10.1038/bjc.2012.127

3. Mitrovic B, Schaeffer DF, Riddell RH, Kirsch R. Tumor budding in colorectal carcinoma: time to take notice. Mod Pathol. 2012; 25 (10): 1315-1325. https://org/10.1038/modpathol.2012.94

4. Zlobec I, Lugli A. Prognostic and predictive factors in colorectal cancer. Postgrad Med J 2008; 84 (994): 403-411. https://org/10.1136/jep.2007.054858

5. van Wyk HC, Park J, Roxburgh C, Horgan P, Foulis A, McMillan DC. The role of tumour budding in predicting survival in patients with primary operable colorectal cancer: a systematic review.

Cancer Treat Rev. 2015; 41 (2): 151- 159. https://org/10.1016/j.ctrv.2014.12.007 
6. Beaton C, Twine CP, Williams GL, Radcliffe AG. Systematic review and meta-analysis of histopathological factors influencing the risk of lymph node metastasis in early colorectal cancer.

Colorectal Dis. 2013; 15 (7): 788- 797.

https://org/10.1111/codi.12129

7. Bosch SL, Teerenstra S, de Wilt JH, Cunningham C, Nagtegaal ID. Predicting lymph node metastasis in pT1 colorectal cancer: a systematic review of risk factors providing rationale for therapy decisions. Endoscopy. 2013; 45 (10): 827-834. https://org/10.1055/s-0033-1344238

8. Cappellesso R, Luchini C, Veronese N, et al. Tumor budding as a risk factor for nodal metastasis in $\mathrm{pT} 1$ colorectal cancers: a metaanalysis. Hum Pathol.2017;65:62-70. https://org/10.1016/j.humpath.2017.04.013

9. Carrara A, Mangiola D, Pertile R, et al. Analysis of risk factors for lymph nodal involvement in early stages of rectal cancer: when can local excision be considered an appropriate treatment? Systematic review and meta-analysis of the literature. Int J Surg Oncol. 2012; 2012: 438450. https://org/10.1155.2012.438450

10. Ishikawa $\mathrm{Y}$, Aishima-Fukasawa $\mathrm{Y}$, Ito $\mathrm{K}$ et al. Histopathological determinants of regional lymph node metastasis in early colorectal cancer. Cancer. 2008; 112 (4): 924-933.

https://doi.org/10.1007/s00428-008-0717-3

11. Choi JY, Jung SA, Shim KN, et al. Meta-analysis of predictive clinicopathologic factors for lymph node metastasis in patients with early colorectal carcinoma. J Korean Med Sci. 2015;30 (4): 398-406. https://org/10.3346/jkms.2015.30.4.398

12. Di Gregorio C, Bonetti LR, de Gaetani C, Pedroni M, Kaleci S, Ponz de Leon M. Clinical outcome of low- and high-risk malignant colorectal polyps: results of a population-based study and meta-analysis of the available literature. Intern Emerg Med. 2014; 9 (2): 151-160. https://org/10.1007/s11739-012-0772-2

13. Glasgow SC, Bleier JI, Burgart LJ, Finne CO, Lowry AC. Metaanalysis of histopathological features of primary colorectal cancers that predict lymph node metastases. J Gastrointest Surg. 2012; 16(5): 1019-1028. https://doi.org/10.1007/s11605-012-1827-4
14. Mou S, Soetikno R, Shimoda T, Rouse R, Kaltenbach T. Pathologic predictive factors for lymph node metastasis in submucosal invasive (T1) colorectal cancer: a systematic review and metaanalysis. Surg Endosc. 2013; 27 (8): 2692- 2703. https://doi.org/10.1007/s00464-013-2835-5

15. Petrelli F, Pezzica E, Cabiddu M, et al. Tumour Budding and Survival in Stage II Colorectal Cancer: a Systematic Review and Pooled Analysis. J Gastrointest Cancer. 2015; 46 (3):212- 218. https://doi.org/10.1007/s12029-015-9716-1

16. Rogers AC, Winter DC, Heeney A, et al. Systematic review and meta-analysis of the impact of tumour budding in colorectal cancer. Br J Cancer. 2016; 115 (7): 831-840. https://doi.org/10.1038/bjc.2016.274

17. Koelzer VH, Zlobec I, Berger MD et al. Tumour budding in colorectal cancer revisited; results of a multicenter interobserver study. Virchows Arch. 2015; 466(5); 485-493. https://doi.org/10.1111/his.13164

18. The Royal College of Pathologists of Australia. Colorectal cancer structured reporting protocol (3rd edition 2016). https://www.rcpa.edu.au

19. Schmoll H J, Van Custem E, Stein A et al. ESMO consensus Guidelines for management of patients with colon \& rectum cancer. A personalized approach to clinical decision making. Ann Oncol. 2012;23 (10): 2479-2516.

https://doi.org/10.1093/annonc/mds236

20. Okuyama T, Oya M, Ishikawa $\mathrm{H}$. Budding as a useful prognostic marker in pT3 well- or moderately differentiated rectal adenocarcinoma. J Surg Oncol. 2003;83 (1): 42-47. https://doi.org/10.1002/jso.10230

21. Nakamura T, Mitomi H, Kanazawa H, Ohkura Y, Watanabe M. Tumour budding as an index to identify high- risk patients with stage II colon cancer. Dis Colon \& Rectum. 2008; 51 (5): 568572. https://doi.org/10.1007/s10350-008-9192-9

22. Lai YH, Wu LC, PS et al. Tumour budding is a reproducible index for risk stratification of patients with stage II colon cancer. Colorectal Dis. 2014; 16(4): 259-264.

https://doi.org/10.1111/codi.12454 